\title{
SETS OF UNIQUENESS AND SETS OF MULTIPLICITY. II
}

\author{
BY \\ R. SALEM
}

1. Introduction. In a recent paper we have investigated the properties of symmetrical perfect sets of constant ratio of dissection from the point of view of their classification in sets of uniqueness and sets of multiplicity for trigonometrical series. (See Salem [1]( $\left.{ }^{1}\right)$, where we refer the reader for the defini tions and the terminology used.) We have shown the connection between this problem and the properties of a class of algebraic integers which we have called "Pisot-Vijayaraghavan numbers," or briefly, "P.V. numbers," and which are defined by the condition that all their conjugates have their moduli inferior to the unity. We have proved that a symmetrical perfect set of constant ratio of dissection $\xi$ is a set of uniqueness if and only if $\xi$ is the reciprocal of a P.V. number. For all other values of $\xi$ the set is a set of multiplicity.

The main purpose of this paper is to study, from the same point of view, sets of constant ratio of dissection, but unsymmetric, and to find the necessary and sufficient conditions for these sets to be sets of uniqueness ( $\$ 2-8)$.

The solution of this problem will lead us to a particular result in the classification of symmetrical perfect sets of the Cantor type and of variable ratio of dissection ( $\$ 9)$. The general problem for such sets remains unsolved, but we shall give results showing some of the features of the problem $(\$ 10-13)$.

2. Definition of unsymmetric perfect sets of constant ratio of dissection. Let $A B$ be an interval of length $L$, let $d$ be an integer not less than 1 , and let us mark, on $A B, d+1$ "white intervals," nonoverlapping, of the same length $L \xi(0<\xi<1 /(d+1))$, the origin of the first interval being $A$ and the extremity of the last interval being $B$. We then remove the $d$ open "black intervals" lying between two consecutive white intervals (some black intervals may be empty if two successive white intervals are abutting). Let $L \alpha_{0}=0, L \alpha_{1}, L \alpha_{2}, \cdots, L \alpha_{d}$ be the distances from $A$ to the origins of the $d+1$ white intervals. (We have $\alpha_{d}=1-\xi$ and $\alpha_{j+1}-\alpha_{j} \geqq \xi$.)

Such a dissection will be called a $\left(d, \xi, \alpha_{1}, \alpha_{2}, \cdots, \alpha_{d}\right)$ dissection of the given interval.

Let us now start with a $\left(d, \xi, \alpha_{1}, \alpha_{2}, \cdots, \alpha_{d}\right)$ dissection of the interval $(0,2 \pi)$ and let us remove the black intervals.

In a second step we make a $\left(d, \xi, \alpha_{1}, \cdots, \alpha_{d}\right)$ dissection of each of the $d+1$ white intervals left after the first dissection, and we remove the black intervals.

Presented to the Society, February 26, 1944; received by the editors December 29, 1943.

1 Numbers in brackets refer to the Bibliography at the end of the paper. 
We proceed in the same way indefinitely and we get thus a perfect set $P$ of measure zero, which we shall call an unsymmetric perfect set of constant ratio of dissection $\left(d, \xi, \alpha_{1}, \cdots, \alpha_{d}\right)$.

It is easily seen that after $p$ dissections we get $(d+1)^{p}$ white intervals, each of length $2 \pi \xi^{p}$, whose left-hand end points have their abscissae given by the sum of $p$ terms

$$
2 \pi\left[\alpha\left(\epsilon_{1}\right)+\alpha\left(\epsilon_{2}\right) \xi+\alpha\left(\epsilon_{3}\right) \xi^{2}+\cdots+\alpha\left(\epsilon_{p}\right) \xi^{p-1}\right],
$$

where $\alpha(j)$ stands for $\alpha_{j}(\alpha(0)=0)$ and where the $\epsilon_{k}$ take all values $0,1,2, \cdots, d$.

Also the points of the perfect set $P$ have their abscissae given by the infinite series

$$
x=2 \pi\left[\alpha\left(\epsilon_{1}\right)+\alpha\left(\epsilon_{2}\right) \xi+\alpha\left(\epsilon_{3}\right) \xi^{2}+\cdots+\alpha\left(\epsilon_{p}\right) \xi^{p-1}+\cdots\right] .
$$

3. Construction of a continuous non-decreasing function constant in each interval contiguous to $P$. To each integer $k$ we associate a set of $d+1$ numbers $\lambda_{k}(0), \lambda_{k}(1), \cdots, \lambda_{k}(d)$ satisfying the following conditions:

(1) they are non-negative,

(2) they are all less than or equal to $\mu_{k}, \mu_{k}$ being inferior to 1 ,

(3) they are such that $\lambda_{k}(0)+\lambda_{k}(1)+\cdots+\lambda_{k}(d)=1$.

Our function $F$ will be the limit, for $p=\infty$, of the function $F_{p}(x)$ defined in the following manner: $F_{p}(0)=0, F_{p}(2 \pi)=1 ; F_{p}(x)$ is continuous and increases linearly by $\lambda_{1}\left(\epsilon_{1}\right) \lambda_{2}\left(\epsilon_{2}\right) \cdots \lambda_{p}\left(\epsilon_{p}\right)$ in the white interval whose left-hand end point is given by (1); finally $F_{p}(x)$ is constant in each black interval.

Obviously

$$
\left|F_{p+1}(x)-F_{p}(x)\right| \leqq \mu_{1} \mu_{2} \cdots \mu_{p} .
$$

Hence if the series $\sum_{1}^{\infty} \mu_{1} \mu_{2} \cdots \mu_{p}$ converges, which we shall assume, $F_{p}(x)$ tends uniformly to a function $F(x)$ continuous, non-decreasing, constant in every interval contiguous to $P$.

4. Fourier-Stieltjes coefficients of $F$. Taking as points of subdivision the ordinates corresponding to the black intervals removed after the $p$ th step of the dissection we obtain for approximate expression of the integral $\int_{0}^{2 \pi} e^{n i x} d F$ :

$$
\begin{aligned}
& \sum \lambda_{1}\left(\epsilon_{1}\right) \lambda_{2}\left(\epsilon_{2}\right) \cdots \lambda_{p}\left(\epsilon_{p}\right) e^{2 \pi n i\left[\alpha\left(\epsilon_{1}\right)+\alpha\left(\epsilon_{2}\right) \xi+\cdots+\alpha\left(\epsilon_{p}\right) \xi^{p-1}\right]} \\
& =\prod_{k=1}^{p}\left[\lambda_{k}(0)+\lambda_{k}(1) e^{2 \pi n i \cdot \alpha(1) \xi^{k-1}}+\lambda_{k}(2) e^{2 \pi n i \cdot \alpha(2) \xi^{k-1}}+\cdots+\lambda_{k}(d) e^{2 \pi n i \cdot \alpha(d) \xi^{k-1}}\right],
\end{aligned}
$$

so that

$$
\int_{0}^{2 \pi} e^{n i x} d F=\prod_{k=1}^{\infty}\left[\lambda_{k}(0)+\lambda_{k}(1) e^{2 \pi n i \alpha(1) \xi^{k-1}}+\cdots+\lambda_{k}(d) e^{2 \pi n i \alpha(d) \xi^{k-1}}\right] .
$$

5. Choice of a particular function $F$. In what follows we shall make use of a particular type of function $F$ obtained by taking, for each integer $k$, 


$$
\lambda_{k}(0)=1 / 2, \quad \lambda_{k}\left(t_{k}\right)=1 / 2,
$$

$t_{k}$ being a function of $k$ taking only the values $1,2, \cdots, d$, and $\lambda_{k}(s)=0$ for $s \neq 0, s \neq t_{k}$.

In this case we have

and

$$
\int_{0}^{2 \pi} e^{n i x} d F=\prod_{k=1}^{\infty}\left[1 / 2+(1 / 2) e^{2 \pi n i \alpha\left(t_{k}\right) \xi^{k-1}}\right]
$$

$$
\left|\int_{0}^{2 \pi} e^{n i x} d F\right|=\prod_{k=1}^{\infty}\left|\cos \pi n \cdot \alpha\left(t_{k}\right) \xi^{k-1}\right|
$$

6. Outline of some previous results. For the classification of our sets we shall make use of the following results which are all proved in Salem [1] and which we summarize here for the convenience of the reader.

If the infinite product

$$
\gamma(u)=\prod_{k=1}^{\infty} \cos \pi u \xi^{k-1}
$$

does not tend to zero as $u \rightarrow \infty$, then $\xi$ is the reciprocal of a P.V. number.

This is proved by showing first that if $\gamma(u) \neq o(1)$, there then exists a number $\lambda$ different from zero such that if $\xi=1 / \theta$ the series

converges.

$$
\sum_{0}^{\infty} \sin ^{2} \pi \lambda \theta^{n}
$$

Denoting by $a_{n}$ the integer nearest to $\lambda \theta^{n}$ and putting $\lambda \theta^{n}=a_{n}+\mu_{n}$ it is then proved, following Pisot, that the convergence of the last series involves that the determinant

$$
D_{n}=\left|\begin{array}{llll}
a_{0} & a_{1} & \cdots & a_{n} \\
a_{1} & a_{2} & \cdots & a_{n+1} \\
\cdot & \cdot & \cdots & \cdot \\
a_{n} & a_{n+1} & \cdots & a_{2 n}
\end{array}\right|
$$

is equal to zero for all $n$ larger than a certain fixed integer. This, by a well known theorem of Kronecker, involves that $\sum_{0}^{\infty} a_{n} z^{n}$ represents a rational function, and by a theorem of Fatou, since the $a_{n}$ are integers, that this rational function is of the form $P(z) / Q(z), P$ and $Q$ being polynomials with integral coefficients and $Q(0)$ being equal to 1 .

From this the result follows quite easily by writing

$$
\sum_{0}^{\infty} \mu_{n} z^{n}=\sum_{0}^{\infty} \lambda \theta^{n} z^{n}-\sum_{0}^{\infty} a_{n} z^{n}=\frac{\lambda}{1-\theta z}-\frac{P(z)}{Q(z)}
$$

and by comparing the singularities of the two members of the equality. It is 
found that $1 / \theta$ is a root of $Q(z)=0$, all the other roots having moduli larger than 1 ; hence $\theta$ is an algebraic integer whose conjugates all lie inside the unit circle, the equation determining $\theta$ being $z^{k} Q(1 / z)=0(k=$ degree of $Q)$.

7. Lemma. We shall need the following lemma which is an easy deduction from the results summarized in the preceding paragraph:

Lemma. $\theta$ being a P.V. number, then, given any $m$ numbers $\alpha_{1}, \alpha_{2}, \cdots, \alpha_{m}$ in the field $K(\theta)$, there are $m$ numbers $\lambda_{1}, \lambda_{2}, \cdots, \lambda_{m}$ proportional to $\alpha_{1}, \alpha_{2}, \cdots, \alpha_{m}$, such that the series $\sum_{0}^{\infty} \sin ^{2} \pi \lambda \theta^{n}$ converges when $\lambda$ is equal to any of the numbers $\lambda_{1}, \lambda_{2}, \cdots, \lambda_{m}$.

In fact it follows from the results of the preceding paragraph that if $\sum_{0}^{\infty} \sin ^{2} \pi \lambda \theta^{n}<\infty, \theta$ is a P.V. number and that, by (2),

$$
\lambda=\lim _{z \rightarrow 1 / \theta}(1-\theta z) P(z) / Q(z) .
$$

Let $\phi(z)=z^{k} Q(1 / z) . \phi(z)=0$ is the equation determining $\theta$. We have $Q(z)$ $=z^{k} \phi(1 / z)$. We can write $P(z)=z^{h} \psi(1 / z)(h=$ degree of $P)$ and we have

$$
\begin{aligned}
\lambda & =\lim _{z \rightarrow 1 / \theta}(1-\theta z) \frac{z^{h} \psi(1 / z)}{z^{k} \phi(1 / z)} \\
& =\lim _{z \rightarrow 1 / \theta}(1 / z-\theta) \frac{z^{h-k+1} \psi(1 / z)}{\phi(1 / z)}=\frac{\psi(\theta)}{\theta^{a} \phi^{\prime}(\theta)},
\end{aligned}
$$

where $\phi^{\prime}(z)$ is the derivative of $\phi(z), \psi(z)$ is a polynomial with integral coefficients, and $q$ is a positive integer. (If $q<0$ we replace the polynomial $\psi(\theta)$ by $\theta^{-q} \psi(\theta)$.)

Conversely, suppose that $\theta$ is a P.V. number, root of $\phi(z)=0$; let $\lambda$ be an algebraic number of the field $K(\theta)$, having the form $\psi(\theta) / \theta^{a} \phi^{\prime}(\theta)$, where $\psi, q$ and $\phi^{\prime}$ have the above signification. Then the series $\sum_{0}^{\infty} \sin ^{2} \pi \lambda \theta^{n}$ is convergent. In fact, we have

$$
\lambda=\frac{\psi(\theta)}{\theta^{q} \phi^{\prime}(\theta)}=\lim _{z \rightarrow 1 / \theta}(1 / z-\theta) \frac{z^{q} \psi(1 / z)}{\phi(1 / z)}=\lim _{z \rightarrow 1 / \theta}(1-\theta z) \cdot z^{q-1} \frac{\psi(1 / z)}{\phi(1 / z)} .
$$

Putting $\psi(1 / z)=z^{-h} P(z), \phi(1 / z)=z^{-k} Q(z)$, where $h$ and $k$ are the degrees of $\psi$ and $\phi$, we have

$$
\lambda=\lim _{z \rightarrow 1 / \theta}(1-\theta z) z^{q-1} z^{k-h} P(z) / Q(z),
$$

where $P$ and $Q$ are polynomials with integral coefficients, and $Q(0)=1$. We remark that, $\theta$ being an algebraic integer of degree $k$, we can always suppose that the degree $h$ of $\psi$ is less than $k$. Hence $k-h-1 \geqq 0$ and since $q \geqq 0$,

$$
\lambda=\lim _{z \rightarrow 1 / \theta}(1-\theta z) P_{1}(z) / Q(z),
$$


$P_{1}(z)$ being a polynomial with integral coefficients. Now the roots of $Q(z)=0$ are $1 / \theta$ and $k-1$ roots of moduli greater than 1 . Hence the function

$$
\lambda /(1-\theta z)-P_{1}(z) / Q(z)
$$

is regular in a circle $|z| \leqq 1+\alpha$, where $\alpha$ is essentially positive. We have

and

$$
\frac{\lambda}{1-\theta z}=\sum_{0}^{\infty} \lambda \theta^{n} z^{n}
$$

$$
\frac{P_{1}(z)}{Q(z)}=\sum_{0}^{\infty} a_{n} z^{n}
$$

where the $a_{n}$ are integers, since $Q(0)=1$, and the coefficients of $P$ and $Q$ are integers. Hence the series $\sum_{0}^{\infty}\left(\lambda \theta^{n}-a_{n}\right)^{2}$ is convergent (and converges like a geometric progression). This proves the convergence of $\sum_{0}^{\infty} \sin ^{2} \pi \lambda \theta^{n}$.

Now, to prove our lemma, let

$$
\alpha_{1}=p_{1}(\theta) / q(\theta), \alpha_{2}=p_{2}(\theta) / q(\theta), \cdots, \alpha_{m}=p_{m}(\theta) / q(\theta)
$$

be the $m$ given numbers of the field $K(\theta)$, where the $p_{i}(\theta)$ and $q(\theta)$ are polynomials with integral coefficients. We can take

$$
\lambda_{1}=p_{1}(\theta) / \phi^{\prime}(\theta), \lambda_{2}=p_{2}(\theta) / \phi^{\prime}(\theta), \cdots, \lambda_{m}=p_{m}(\theta) / \phi^{\prime}(\theta),
$$

which proves the lemma.

We add two remarks, which will be useful later:

Remark 1. We can take for the $\lambda_{i}$, instead of the preceding values, $\lambda_{i}=p_{i}(\theta) / \theta^{a} \phi^{\prime}(\theta)(i=1,2, \cdots, m), q$ being any positive integer. Hence we can choose the $\lambda_{i}$ such that $\left|\lambda_{i}\right|<\alpha(i=1,2, \cdots, m), \alpha$ being any fixed number.

Remark 2. Since each of the series $\sum_{0}^{\infty} \sin ^{2} \pi \lambda_{i} \theta^{n}(i=1,2, \cdots, m)$ converges like a geometric progression, there is a convergent geometric progression dominating the $m$ series $\sum_{0}^{\infty} \sin ^{2} \pi \lambda_{i} \theta^{n}$. In other words, there exists a positive $\delta<1$, and a positive constant $A$ such that $\left|\left\{\lambda_{i} \theta^{n}\right\}\right|<A \delta^{n}$ $(n=1,2, \cdots)$ for $i=1,2, \cdots, m$, where $\{u\}$ denotes, as usual, the difference between $u$ and the nearest integer.

8. The main theorem. We can now prove our main theorem.

THEOREM I. If the unsymmetric perfect set $P$ of constant ratio of dissection $\left(d, \xi, \alpha_{1}, \cdots, \alpha_{d}\right)$ is a set of uniqueness then

1. $\theta=1 / \xi$ is a P.V. number,

2. $\alpha_{1}, \alpha_{2}, \cdots, \alpha_{d}$ are algebraic numbers of the field $K(\theta)$.

Conversely, if these two conditions are satisfied, $P$ is a set of uniqueness.

Suppose first that $P$ is a set of uniqueness. Let us choose our function $F$ defined in $\$ 5$ by taking $t_{k}$ independent of $k$ and always equal to $d$. Then 


$$
\left|\int_{0}^{2 \pi} e^{n i x} d F\right|=\left|\prod_{k=1}^{\infty} \cos \pi n(1-\xi) \xi^{k-1}\right| .
$$

If this expression does not tend to zero as $n \rightarrow \infty$, it follows from $\S 6$ that $1 / \xi$ is a P.V. number. This is a trivial consequence of the results of $\$ 6$, which can also be obtained by observing that $P$ contains a symmetrical perfect set of constant ratio of dissection $\xi$, and that a subset of a set of uniqueness is also a set of uniqueness.

Let us now choose our function $F$ by taking $t_{k}$, for every $k$, such that

$$
k-t_{k} \equiv 0(\bmod d) \text {. }
$$

In other words $\alpha\left(t_{k}\right)$ is $\alpha_{1}$ when $k \equiv 1(\bmod d), \alpha\left(t_{k}\right)$ is $\alpha_{2}$ when $k \equiv 2(\bmod d)$ $\cdots$ and $\alpha\left(t_{k}\right)$ is $\alpha_{d}$ when $k \equiv d(\bmod d)$. From

$$
\left|\int_{0}^{2 \pi} e^{n i x} d F\right|=\prod_{k=1}^{\infty}\left|\cos \pi n \alpha\left(t_{k}\right) \xi^{k-1}\right|
$$

we deduce that if $P$ is a set of uniqueness, then for an infinite sequence of values of $n: n_{1}, n_{2}, \cdots, n_{s}, \cdots$, the $d$ inequalities

$$
\begin{aligned}
& \left|\cos \pi n \alpha_{1} \cdot \cos \pi n \alpha_{1} \xi^{d} \cdot \cos \pi n \alpha_{1} \xi^{2 d} \ldots\right|>a, \\
& \left|\cos \pi n \alpha_{2} \xi \cdot \cos \pi n \alpha_{2} \xi^{d+1} \cdot \cos \pi n \alpha_{2} \xi^{2 d+1} \ldots\right|>a, \\
& \left|\cos \pi n \alpha_{d} \xi^{d-1} \cdot \cos \pi n \alpha_{d} \xi^{2 d-1} \cdot \cos \pi n \alpha_{d} \xi^{3 d-1} \ldots\right|>a,
\end{aligned}
$$

$a$ being a positive constant, hold together.

Let us write

and let

$$
\alpha_{i} \xi^{i-1}=\beta_{i} \quad(i=1,2, \cdots, d)
$$

$$
\xi=1 / \theta, \quad \theta^{d}=\Theta .
$$

We have when $n$ belongs to the sequence $\left\{n_{s}\right\}$ :

$$
\left|\cos \pi n \beta_{i} \cdot \cos \pi n \beta_{i} \xi^{d} \cdot \cos \pi n \beta_{i} \xi^{2 d} \ldots\right|>a \quad(i=1,2, \cdots, d) .
$$

Let

$$
\Theta^{m-1}<n_{s} \leqq \Theta^{m},
$$

the integer $m=m$, being a function of $s$. We can write $n_{s}=\mu_{s} \Theta^{m}$ where $1 / \Theta<\mu_{s} \leqq 1$. Let us take an infinite subsequence $\left\{m_{q}\right\}$ of $\left\{m_{s}\right\}$ such that $\mu_{q}$ tends to a limit $\mu$ as $q \rightarrow \infty$. Taking $n=\mu_{q} \Theta^{m_{q}}$ we have

$$
\left|\cos \pi \mu_{q} \beta_{i} \cos \pi \mu_{q} \beta_{i} \Theta \cdots \cos \pi \mu_{q} \beta_{i} \Theta^{m_{q}}\right|>a \quad(i=1,2, \cdots, d) .
$$

From this we deduce easily (the details of the calculations, which are obvious, can be found in Salem [1, p. 222]) 


$$
\begin{aligned}
\sin ^{2} \pi \mu_{q} \beta_{i}+\sin ^{2} \pi \mu_{q} \beta_{i} \Theta+\cdots+\sin ^{2} \pi \mu_{q} \beta_{i} \Theta^{m_{q}} \leqq & \log \left(1 / a^{2}\right) \\
& (i=1,2, \cdots, d) .
\end{aligned}
$$

In the same way, taking $n=\mu_{r} \Theta^{m_{r}}(r>q)$ and $m_{r}$ belonging to the sequence $\left\{m_{q}\right\}$, we have

$$
\begin{aligned}
\sin ^{2} \pi \mu_{r} \beta_{i}+\sin ^{2} \pi \mu_{r} \beta_{i} \Theta+\cdots+\sin ^{2} \pi \mu_{r} \beta_{i} \Theta^{m_{r}} \leqq & \log \left(1 / a^{2}\right) \\
& (i=1,2, \cdots, d),
\end{aligned}
$$

and since $r>q$, we have a fortiori

$$
\begin{aligned}
\sin ^{2} \pi \mu_{r} \beta_{i}+\sin ^{2} \pi \mu_{r} \beta_{i} \Theta+\cdots+\sin ^{2} \pi \mu_{r} \beta_{i} \Theta^{m_{q}} \leqq & \log \left(1 / a^{2}\right) \\
& (i=1,2, \cdots, d) .
\end{aligned}
$$

Keeping $q$ fixed and letting $r \rightarrow \infty$ we have, as $\mu_{r} \rightarrow \mu$,

$$
\sin ^{2} \pi \mu \beta_{i}+\sin ^{2} \pi \mu \beta_{i} \Theta+\cdots+\sin ^{2} \pi \mu \beta_{i} \Theta{ }^{m_{r}} \leqq \log \left(1 / a^{2}\right)
$$

and, since $m_{q}$ is arbitrarily large,

$$
(i=1,2, \cdots, d)
$$

$$
\sum_{0}^{\infty} \sin ^{2} \pi \mu \beta_{i} \Theta^{m}<\infty \quad(i=1,2, \cdots, d) .
$$

We know (see $\$ \S 6$ and 7 ) that this involves that the numbers $\mu \beta_{i}$ are all algebraic numbers of the field $K(\Theta)$, hence also of the field $K(\theta)$. The same is true for the numbers $\mu \alpha_{i}$. Hence $\alpha_{1}, \alpha_{2}, \cdots, \alpha_{d}$ are proportional to algebraic numbers of the field $K(\theta)$. But since $\alpha_{d}=1-\xi=1-1 / \theta$, the numbers $\alpha_{1}, \alpha_{2}, \cdots, \alpha_{d}$ belong to the field $K(\theta)$. This proves the first part of the theorem.

To prove the second part we suppose that $\theta=1 / \xi$ is a P.V. number and that $\alpha_{1}, \alpha_{2}, \cdots, \alpha_{d}$ belong to the field $K(\theta)$.

Then by the lemma we can find $d$ numbers $\lambda_{i}(i=1,2, \cdots, d)$ proportional to the $\alpha_{i}$ and such that

$$
\sum_{0}^{\infty} \sin ^{2} \pi \lambda_{i} \theta^{n}<c \quad \text { for } i=1,2, \cdots, d .
$$

Let $\lambda_{i}=\lambda \alpha_{i}(\lambda>0)$. Then

$$
\sum_{0}^{\infty} \sin ^{2} \pi \lambda \alpha_{i} \theta^{n}<\infty \quad \text { for } i=1,2, \cdots, d .
$$

By the first remark of $\S 7$, we can suppose $\lambda<\theta$ and by the second remark we can find a positive number $\delta<1$ and a constant $A$ such that, for all $n$,

$$
\left|\left\{\lambda \alpha_{i} \theta^{n}\right\}\right|<A \delta^{n}, \quad i=1,2, \cdots, d,
$$

where $\{u\}$ denotes the difference between $u$ and the nearest integer. 
We can now prove that the set $P$ is a set of uniqueness. The argument, although a little less simple, is in essentials the same as in Salem [1].

The points of $P$ are given by

$$
\begin{aligned}
x & =2 \pi\left[\alpha\left(\epsilon_{1}\right)+\alpha\left(\epsilon_{2}\right) \xi+\cdots+\alpha\left(\epsilon_{p}\right) \xi^{p-1}+\cdots\right] \\
& =2 \pi \theta\left[\alpha\left(\epsilon_{1}\right) / \theta+\alpha\left(\epsilon_{2}\right) / \theta^{2}+\cdots+\alpha\left(\epsilon_{p}\right) / \theta^{p}+\cdots\right],
\end{aligned}
$$

where the $\epsilon_{i}$ take the values $0,1, \cdots, d$. Let

$$
y=(\lambda / \theta) x \text {. }
$$

Since $\lambda<\theta$ the set $G$ of the points $y$ lies in $(0,2 \pi)$ like the set $P$ of the points $x$. We have

$$
y=2 \pi \lambda\left[\alpha\left(\epsilon_{1}\right) / \theta+\alpha\left(\epsilon_{2}\right) / \theta^{2}+\cdots\right] .
$$

Let $h$ be a fixed integer to be chosen later and consider the $(d+1)^{h}$ possible combinations of $\epsilon_{1} \epsilon_{2} \cdots \epsilon_{h}$ where $\epsilon_{i}=0,1, \cdots, d$. Let $C_{1} C_{2} \cdots C_{(d+1)}{ }^{h}$ denote the combinations. Writing

(4) $y=2 \pi \lambda\left[\left(\alpha\left(\epsilon_{1}\right) / \theta+\cdots+\alpha\left(\epsilon_{h}\right) / \theta^{h}\right)+\left(\alpha\left(\epsilon_{h+1}\right) / \theta^{h+1}+\cdots+\alpha\left(\epsilon_{2 h}\right) / \theta^{2 h}\right)+\cdots\right]$, consider the sequence of the $\epsilon_{i}$ belonging to one same group of terms in parenthesis. They form one of the $(d+1)^{h}$ combinations $C_{1} C_{2} \cdots C_{(d+1)^{h}}$.

Denote by $G_{s}\left(s=1,2, \cdots,(d+1)^{h}\right)$ the subset of $G$ such that if $y$ belongs to $G_{s}$ the combination $C_{s}$ occurs in infinitely many groups of terms in parenthesis when $y$ is written in the form (4). Then

$$
G=G_{1}+G_{2}+\cdots+G_{(d+1)^{h}} .
$$

$G$ being closed we have also

$$
G=\left(G_{1}+G_{1}^{\prime}\right)+\left(G_{2}+G_{2}^{\prime}\right)+\cdots+\left(G_{(d+1)^{h}}+G_{(d+1)^{h}}^{\prime}\right),
$$

where $G_{s}^{\prime}$ denotes the derived set of $G_{s}$.

Considering now $G_{s}$ for a fixed $s$ let $m_{1}, m_{2}, \cdots, m_{n}, \cdots$ be the infinite sequence of the ranks of the groups of terms in parenthesis where the combination $C_{s}$ occurs. Let $z$ be any point belonging to $G_{s}$. We have

$$
\begin{aligned}
z \theta^{h m_{n}}= & 2 \pi \lambda\left[\alpha\left(\epsilon_{h m_{n}}\right)+\theta \alpha\left(\epsilon_{h m_{n}-1}\right)+\cdots+\theta^{h-1} \alpha\left(\epsilon_{h m_{n}-h+1}\right)\right] \\
& +2 \pi \lambda\left[\theta^{h} \alpha\left(\epsilon_{h m_{n}-h}\right)+\cdots+\theta^{h m_{n}-1} \alpha\left(\epsilon_{1}\right)\right] \\
& +2 \pi \lambda\left[\frac{\alpha\left(\epsilon_{h m_{n}+1}\right)}{\theta}+\frac{\alpha\left(\epsilon_{h m_{n}+2}\right)}{\theta^{2}}+\cdots\right],
\end{aligned}
$$

and denoting the three successive brackets by $\beta(s), \gamma(z), y(z)$ respectively we have

$$
z \theta^{h m_{n}}=\beta(s)+\gamma(z)+y(z),
$$

where $\gamma(z)$ and $y(z)$ depend on the particular point $z$, but $\beta(s)$ depends on $s$ only but not on $z$. 
Let us put

$$
\lambda \alpha\left(\epsilon_{h m_{n}-k}\right) \theta^{k}=a_{k}+\mu_{k} \quad\left(k=h, h+1, \cdots, h m_{n}-1\right),
$$

where $a_{k}$ is the integer nearest to the first member of the equality. We have

$$
\begin{aligned}
\gamma(z) & =2 \pi\left[a_{h}+a_{h+1}+\cdots+a_{h m_{n}-1}\right]+2 \pi\left[\mu_{h}+\mu_{h+1}+\cdots+\mu_{h m_{n}-1}\right] \\
& =2 \pi N(z)+\gamma_{1}(z),
\end{aligned}
$$

say, $N(z)$ being an integer. But by (3)

$$
\left|\mu_{k}\right|<A \delta^{k}
$$

hence

$$
\left|\gamma_{1}(z)\right|<2 \pi A\left(\delta^{h}+\delta^{h+1}+\cdots\right)=2 \pi A \delta^{h} /(1-\delta) .
$$

Also, since $\theta$ is a P.V. number, we have, $b_{h m_{n}}$ being the integer nearest to $\theta^{h m_{n}}$,

$$
\theta^{h m_{n}}=b_{h m_{n}}+\left\{\theta^{h m_{n}}\right\}
$$

and, for $m_{n}$ large enough,

$$
\left|\left\{\theta^{h m_{n}}\right\}\right|<\delta^{\prime h m_{n}}
$$

where $\delta^{\prime}<1$. Hence

$$
z \theta^{h m_{n}}=z b_{h m_{n}}+z\left\{\theta^{h m_{n}}\right\}
$$

where

$$
\left|z\left\{\theta^{h m_{n}}\right\}\right|<2 \pi \delta^{\prime h} \text {. }
$$

Finally, we remark that $y(z)$ is a point belonging to $G_{\bullet}$, hence to $G$.

Let $l$ be the length of the greatest interval contiguous to $G$, and let us suppose that $h$ has been chosen such that the second members of (5) and (6) be both less than $l / 8$. Then we have

$$
z b_{h m_{n}}=2 \pi N(z)+\beta(s)+y(z)+\gamma_{2}(z),
$$

where

$$
\gamma_{2}(z)=\gamma_{1}(z)-z\left\{\theta^{h m_{n}}\right\}, \quad\left|\gamma_{2}(z)\right|<l / 4 .
$$

This is equivalent to

$$
z b_{h m_{n}} \equiv \beta(s)+y(z)+\gamma_{2}(z)
$$

From now on, the proof follows exactly the argument given in Salem [1] for symmetrical perfect sets of constant ratio. We repeat it briefly: the last equality proves that the set $G_{s}^{n}$ deduced from $G$, by multiplication of $z$ by the integer $b_{h m_{n}}$ and reduction modulo $2 \pi$ has a contiguous interval of length not less than $l-2 l / 4=l / 2$. The same is true for the closed set $G_{s}^{n}+\left(G_{s}^{n}\right)^{\prime}$, 
which proves that the set $G_{\bullet}+G_{\bullet}^{\prime}$ is of the type $H$. Hence the set

$$
G=\sum_{1}^{(d+1)^{n}}\left(G_{\imath}+G_{\imath}^{\prime}\right) \text {, }
$$

being the sum of closed sets of the type $H$, is a set of uniqueness.

Finally $P$ being homothetic of $G$, and both sets lying in $(0,2 \pi)$, is also a set of uniqueness, which achieves the proof of our theorem.

9. Application to some symmetrical perfect sets of the Cantor type and variable ratio of dissection. We denote by this expression a perfect set obtained by a trisection of the fundamental interval $(0,2 \pi)$ in three parts of lengths proportional to $\xi_{1}, 1-2 \xi_{1}, \xi_{1}$, respectively, and the removal of the central "black" interval. Each "white interval" left is trisected in parts proportional to $\xi_{2}, 1-2 \xi_{2}, \xi_{2}$, and the two central intervals are removed. The process is continued indefinitely, the sequence $\xi_{1}, \xi_{2}, \ldots, \xi_{p}, \ldots$ being such that $0<\xi_{p}<1 / 2$. If $2^{p} \xi_{1} \xi_{2} \cdots \xi_{p} \rightarrow 0$, the perfect set obtained has measure zero. The points of the set are given by the formula

$$
x=2 \pi\left[\epsilon_{1}\left(1-\xi_{1}\right)+\epsilon_{2} \xi_{1}\left(1-\xi_{2}\right)+\cdots+\epsilon_{p} \xi_{1} \cdots \xi_{p-1}\left(1-\xi_{p}\right)+\cdots\right],
$$

where the $\epsilon_{i}$ are 0 or 1 .

The problem of finding the necessary and sufficient conditions which must be satisfied by the sequence $\left\{\xi_{p}\right\}$ in order that the set be a set of uniqueness is unsolved. We shall deal here with a particular case as an application of the theory previously developed for unsymmetric perfect sets of constant ratio.

We shall suppose henceforth that we are given $g$ numbers $\xi_{1}, \xi_{2}, \cdots, \xi_{0}$ and that

$$
\xi_{p}=\xi_{i} \quad \text { if } \quad p \equiv i(\bmod g) \quad(i=1,2, \cdots, g),
$$

in other words, we have a periodicity of the ratios of dissection, the first $g$ ratios being arbitrary.

Let

$$
\xi_{1} \xi_{2} \cdots \xi_{0}=X
$$

and let

$$
r_{1}=1-\xi_{1}, r_{2}=\xi_{1}\left(1-\xi_{2}\right), \cdots, r_{0}=\xi_{1} \cdots \xi_{0-1}\left(1-\xi_{g}\right) .
$$

Thus

$$
\begin{aligned}
x=2 \pi\left[\left(\epsilon_{1} r_{1}+\epsilon_{2} r_{2}+\cdots+\epsilon_{g} r_{g}\right)+X\right. & \left(\epsilon_{g+1} r_{1}+\cdots+\epsilon_{2 g} r_{g}\right) \\
& \left.+X^{2}\left(\epsilon_{2 g+1} r_{1}+\cdots+\epsilon_{3 g} r_{g}\right)+\cdots\right] .
\end{aligned}
$$

Now let us observe that

$$
\epsilon_{1} r_{1}+\epsilon_{2} r_{2}+\cdots+\epsilon_{g} r_{g},
$$

where $\epsilon_{i}=0$ or 1 , takes $2^{o}=d+1$ values which we can denote in ascending 
order of magnitude by $\alpha(0), \alpha(1), \cdots, \alpha(d)\left(\alpha(0)=0, \alpha(d)=r_{1}+r_{2}+\cdots+r_{o}\right.$ $=1-X)$. Thus

$$
x=2 \pi\left[\alpha\left(\eta_{1}\right)+\alpha\left(\eta_{2}\right) X+\alpha\left(\eta_{3}\right) X^{2}+\cdots\right],
$$

where $\eta_{i}=0,1, \cdots, d$. We have $X<1 / 2^{o}=1 /(d+1)$. We see that we have here a particular case of an unsymmetric perfect set of constant ratio of dissection and of the type $\left(d, X, \alpha_{1} \alpha_{2} \cdots \alpha_{d}\right)$.

Thus if the set is a set of uniqueness:

1. $X=\xi_{1} \xi_{2} \cdots \xi_{0}$ must be the reciprocal of a $P$.V. number $\theta=1 / X$.

2. The $2^{a}-1$ numbers $\sum_{1}^{0} \epsilon_{i} r_{i}$ (the combination $\epsilon_{1}=\epsilon_{2}=\cdots=\epsilon_{g}=0$ excluded) must be algebraic numbers of the field $K(\theta)$.

Conversely, if these two conditions are satisfied the set is a set of uniqueness.

Now it is obvious that we can replace the condition 2 by the following one:

The numbers $r_{1}, r_{2}, \cdots, r_{0}$ given by (7) must be algebraic numbers of the field $K(\theta)$. (This gives explicitly the admissible values for $\xi_{1}, \xi_{2}, \cdots, \xi_{\theta}$, keeping in mind that $r_{1}+r_{2}+\cdots+r_{0}$ must be equal to $1-X$.)

10. A theorem on general symmetrical perfect sets of the Cantor type. The problem of the general symmetrical perfect sets of the Cantor type when the sequence $\xi_{1} \xi_{2} \cdots \xi_{p} \cdots$ is arbitrary seems difficult. We shall prove the following theorem:

THEOREM. If $\xi_{p}$ has a limit $\xi \neq 0$ as $p \rightarrow \infty$ and if the set is a set of uniqueness, then $1 / \xi$ is necessarily a P.V. number.

Let us consider the function $F(x)$, continuous, non-decreasing, constant in each interval contiguous to the set, and equal to

$$
\epsilon_{1} / 2+\epsilon_{2} / 2^{2}+\cdots+\epsilon_{p} / 2^{p}+\cdots
$$

when $x$ belongs to the set and is given by

$$
x=2 \pi\left[\epsilon_{1}\left(1-\xi_{1}\right)+\epsilon_{2} \xi_{1}\left(1-\xi_{2}\right)+\cdots+\epsilon_{p} \xi_{1} \cdots \xi_{p-1}\left(1-\xi_{p}\right)+\cdots\right]
$$

$\left(\epsilon_{i}=0\right.$ or 1$)$. Then it is easily seen that

$$
\left|\int_{0}^{2 \pi} e^{n i x} d F\right|=\prod_{k=1}^{\infty}\left|\cos \pi n \xi_{1} \cdots \xi_{k-1}\left(1-\xi_{k}\right)\right| .
$$

We suppose $\xi_{k}=\xi\left(1+u_{k}\right)$ where $u_{k} \rightarrow 0$ as $k \rightarrow \infty$. Then

say.

$$
\begin{aligned}
\xi_{1} \cdots \xi_{k-1}\left(1-\xi_{k}\right) & =\xi^{k-1}\left(1+u_{1}\right) \cdots\left(1+u_{k-1}\right)\left(1-\xi-\xi u_{k}\right) \\
& =\xi^{k-1} \phi(k-1),
\end{aligned}
$$

Then

$$
\left|\int_{0}^{2 \pi} e^{n i x} d F\right|=\prod_{k=0}^{\infty}\left|\cos \pi n \phi(k) \xi^{k}\right|
$$


We observe that $\xi_{1} \cdots \xi_{k-1}\left(1-\xi_{k}\right)>\xi_{1} \cdots \xi_{k}\left(1-\xi_{k+1}\right)$ as a result of $1-\xi_{k}$ $>\xi_{k}\left(1-\xi_{k+1}\right)$ which is true since $\xi_{k}<1 / 2$. Hence $\phi(k) \xi^{k}$ is decreasing and tending to zero as $k \rightarrow \infty$.

If the set is a set of uniqueness the Fourier-Stieltjes coefficient of $F$ does not tend to zero. Hence we can find an infinite sequence $\left\{n_{s}\right\}$ such that for $n=n_{s}$

$$
\prod_{k=0}^{\infty}\left|\cos \pi n \phi(k) \xi^{k}\right|>a,
$$

$a$ being a positive constant.

Let $\xi=1 / \theta$; since $\theta^{k} / \phi(k)$ increases infinitely with $k$, we can associate to every $n_{s}$ an integer $m=m_{s}$ such that

$$
\theta^{m-1} / \phi(m-1)<n_{\varepsilon} \leqq \theta^{m} / \phi(m) .
$$

We write

$$
n_{s}=\lambda_{s} \theta^{m_{s}} / \phi\left(m_{s}\right)
$$

and we have $\lambda_{s} \leqq 1$. On the other hand

$$
\lambda_{s}>\left(\theta^{m_{s}-1} / \phi\left(m_{s}-1\right)\right) \cdot\left(\phi\left(m_{s}\right) / \theta^{m_{s}}\right)=(1 / \theta)\left(\phi\left(m_{s}\right) / \phi\left(m_{s}-1\right)\right)
$$

and since $\phi(m) / \phi(m-1)$ approaches 1 as $m \rightarrow \infty$ we have for $s$ large enough

$$
\lambda_{s}>1 / 2 \theta,
$$

hence the sequence $\left\{\lambda_{s}\right\}$ has at least one limiting point $\lambda \neq 0$. Let us consider a subsequence $\left\{m_{q}\right\}$ of the sequence $\left\{m_{s}\right\}$ such that $\lambda_{q} \rightarrow \lambda$. We have, taking $n=n_{q}$,

$$
\begin{aligned}
a^{2}< & \prod_{k=0}^{m_{q}}\left|\cos \left(\pi \lambda_{q}\left(\theta^{m_{q}} / \phi\left(m_{q}\right)\right) \cdot\left(\phi(k) / \theta^{k}\right)\right)\right|^{2} \\
< & \cos ^{2} \pi \lambda_{q} \cdot \cos ^{2} \pi \lambda_{q}\left(\phi\left(m_{q}-1\right) / \phi\left(m_{q}\right)\right) \theta \\
& \cdot \cos ^{2} \pi \lambda_{q}\left(\phi\left(m_{q}-2\right) / \phi\left(m_{q}\right)\right) \theta^{2} \cdots \cos ^{2} \pi \lambda_{q}\left(\phi\left(m_{q}-p\right) / \phi\left(m_{q}\right)\right) \theta^{p}
\end{aligned}
$$

for any integer $p \leqq m_{q}$. From this we deduce easily

$$
\begin{aligned}
\sin ^{2} \pi \lambda_{q} & +\sin ^{2} \pi \lambda_{q}\left(\phi\left(m_{q}-1\right) / \phi\left(m_{q}\right)\right) \theta+\cdots \\
& +\sin ^{2} \pi \lambda_{q}\left(\phi\left(m_{q}-p\right) / \phi\left(m_{q}\right)\right) \theta^{p} \leqq \log \left(1 / a^{2}\right) .
\end{aligned}
$$

Now

$$
\begin{aligned}
\mid \sin ^{2} \pi \lambda_{q}\left(\phi\left(m_{q}-k\right) / \phi\left(m_{q}\right)\right) \theta^{k}-\sin ^{2} & \pi \lambda_{q} \theta^{k} \mid \\
& <\pi \lambda_{q} \theta^{k}\left|\phi\left(m_{q}-k\right) / \phi\left(m_{q}\right)-1\right| .
\end{aligned}
$$

Since $\phi(m) / \phi(m-1) \rightarrow 1$ as $m \rightarrow \infty$, we can write

$$
\phi(m)=\left(1+v_{m}\right) \phi(m-1)
$$

where $v_{m} \rightarrow 0$ as $m \rightarrow \infty$. Then 


$$
\phi(m)=\left(1+v_{m}\right)\left(1+v_{m-1}\right)\left(1+v_{m-2}\right) \cdots\left(1+v_{m-k+1}\right) \phi(m-k) .
$$

Suppose that

$$
\max \left(\left|v_{m}\right|,\left|v_{m-1}\right|, \cdots,\left|v_{m-k+1}\right|\right)=w(m, k)=w
$$

then

$$
(1-w)^{k} \leqq \phi(m) / \phi(m-k) \leqq(1+w)^{k} .
$$

Let now $\omega(m)$ be an integer, function of $m$, increasing infinitely with $m$, and such that $\omega(m)=o(m)$. We have, if $k \leqq \omega(m)$,

$$
w(m, k) \leqq w(m, \omega(m)) \leqq w(m,[m / 2])
$$

say, for $m$ large enough, $[m / 2]$ being the integral part of $m / 2$. This last number tends to zero as $m \rightarrow \infty$; hence taking $\omega(m)$ such that

$$
\omega(m) \cdot w(m,[m / 2]) \rightarrow 0
$$

as $m \rightarrow \infty$, we see that

$$
|\phi(m) / \phi(m-k)-1|
$$

tends uniformly to zero with $m$ for all $k$ such that $1 \leqq k \leqq \omega(m)$. We write, in these conditions,

$$
|\phi(m-k) / \phi(m)-1|<\epsilon(m)
$$

and we can assume that $\epsilon(m)$ tends to zero monotonically. We take in (8) $p<\omega\left(m_{q}\right)$ and we have, by (9),

$$
\left|\sin ^{2} \pi \lambda_{q} \frac{\phi\left(m_{q}-k\right)}{\phi\left(m_{q}\right)} \theta^{k}-\sin ^{2} \pi \lambda_{q} \theta^{k}\right|<\pi \lambda_{q} \theta^{k} \epsilon\left(m_{q}\right) \leqq \pi \theta^{k} \epsilon\left(m_{q}\right)
$$

for $1 \leqq k \leqq p$.

Now choose $p<\omega\left(m_{q}\right)$ such that $p$ is the largest integer such that

$$
\pi p \theta^{p} \epsilon\left(m_{q}\right)<1 .
$$

Since $\epsilon\left(m_{q}\right) \rightarrow 0$ steadily, $p$ is a function of $m_{q}$ increasing infinitely with $m_{q}$. We denote it by $p_{q}$.

Comparing (8), (9), and (10), we have

$$
\sin ^{2} \pi \lambda_{q}+\sin ^{2} \pi \lambda_{q} \theta+\sin ^{2} \pi \lambda_{q} \theta^{2}+\cdots+\sin ^{2} \pi \lambda_{q} \theta^{p_{q}} \leqq \log \left(1 / a^{2}\right)+1 .
$$

In the same way, taking instead of $m_{q}$ an integer $m_{r}$ of the sequence $\left\{m_{q}\right\}$ with $m_{r}>m_{q}$, we have

$$
\sin ^{2} \pi \lambda_{r}+\sin ^{2} \pi \lambda_{r} \theta+\cdots+\sin ^{2} \pi \lambda_{r} \theta^{p_{r}} \leqq \log \left(1 / a^{2}\right)+1
$$

and, since $p_{r} \geqq p_{q}$,

$$
\sin ^{2} \pi \lambda_{r}+\sin ^{2} \pi \lambda_{r} \theta+\cdots+\sin ^{2} \pi \lambda_{r} \theta^{p_{q}} \leqq \log \left(1 / a^{2}\right)+1
$$


Keeping now $q$ fixed, we let $r \rightarrow \infty$, and we get

$$
\sin ^{2} \pi \lambda+\sin ^{2} \pi \lambda \theta+\cdots+\sin ^{2} \pi \lambda \theta^{p_{q}} \leqq \log \left(1 / a^{2}\right)+1 .
$$

Since $p_{q}$ is arbitrarily large, this is equivalent to

$$
\sum_{0}^{\infty} \sin ^{2} \pi \lambda \theta^{n}<\infty,
$$

which proves that $\theta$ is a $P . V$. number.

11. Questions of stability. The conclusion of the preceding theorem is that if $\xi_{k}=\xi\left(1+u_{k}\right), u_{k} \rightarrow 0$, then the set is a set of multiplicity whatever the sequence $\left\{u_{k}\right\}$, if $\xi$ is not the reciprocal of a P.V. number $(\xi \neq 0)$.

On the other hand if $1 / \xi=\theta$ is a P.V. number, a set for which

$$
\xi_{k}=\xi\left(1+u_{k}\right), \quad u_{k} \rightarrow 0,
$$

is not necessarily a set of uniqueness. As a matter of fact, it is, in general, a set of multiplicity. It has been proved, in fact (in Salem [2]), that, given a positive decreasing sequence $\left\{u_{k}\right\}$, if we consider all sets such that

$$
\left(1-u_{k}\right) \xi<\xi_{k}<\left(1+u_{k}\right) \xi
$$

"almost all" of them are sets of multiplicity, provided that $u_{k}$ does not tend to zero too rapidly, more precisely, provided that $\log \left(1 / u_{k}\right)=o(k)$.

A symmetrical perfect set $Q$ such that its $\xi_{k}$ 's satisfy (11) with $u_{k} \rightarrow 0$ can be said to be "in the neighborhood" of the set $P$ for which $\xi_{k}$ is constant and equal to $\xi$. Thus if $P$ is a set of multiplicity, all sets in the neighborhood are also sets of multiplicity; while if $P$ is a set of uniqueness there are sets $Q$ in the neighborhood of $P$ which are sets of multiplicity. This suggests, to use an expression of A. Zygmund, a "stability" of sets of multiplicity, and an "unstability" of sets of uniqueness thus considered.

It would be interesting to investigate if the "stability" of sets of multiplicity is true in more general cases. Let $\xi_{1}^{0}, \xi_{2}^{0}, \cdots, \xi_{k}^{0}, \cdots$ define a set of multiplicity. Let a positive $u_{k} \rightarrow 0$. Is it true that all sets such that

$$
\left(1-u_{k}\right) \xi_{k}^{0}<\xi_{k}<\left(1+u_{k}\right) \xi_{k}^{0}
$$

are sets of multiplicity? We have proved this to be true if $\xi_{k}^{0}=\xi(k=1,2, \cdots)$ but $I$ have not succeeded in proving it in the general case.

Another interesting aspect of the problem of stability is supplied by the symmetrical perfect sets $S$ of Cantor type and of constant ratio of dissection $\xi$, which are sets of uniqueness if and only if $\xi$ is the reciprocal of a P.V. number. It has been proved (Salem [3]) that the set of P.V. numbers is closed, and thus that small variations of $\xi$ will change the character of a set $S$ if it is a set of uniqueness, but not if it is a set of multiplicity. No simple general result, however, can be expected in this direction, for certain sets of multiplicity can also be, in this last sense, unstable. Consider the sym- 
metrical perfect set of Cantor type where $\xi_{i}$ is equal to $\xi$ or to $\eta$ according as $i$ is odd or even. Let $\xi \eta=1 / \theta$, where $\theta$ is a P.V. number, but suppose that $\xi$ (and so $\eta$ ) are transcendental. Then the set is of multiplicity but can be transformed into a set of uniqueness by changing $\xi$ and $\eta$ as little as we please if we make them equal to algebraic numbers of the field $K(\theta)$. (See $\S 9$.)

12. Example of a set of uniqueness with transcendental ratios of dissection. We have mentioned that the problem of classification of general symmetrical perfect sets of the Cantor type seems difficult. We shall give an example to illustrate the complication of the cases which may arise.

Let, according to $\S 9, \xi_{1} \xi_{2} \cdots \xi_{p} \cdots\left(\xi_{p}<1 / 2\right)$ be the sequence defining the set $P$. We write

$$
r_{1}=1-\xi_{1}, r_{2}=\xi_{1}\left(1-\xi_{2}\right), \cdots, r_{k}=\xi_{1} \cdots \xi_{k-1}\left(1-\xi_{k}\right), \cdots .
$$

It is easy to see that the $r_{k}>0$ are arbitrary, except for the following conditions,

$$
\begin{gathered}
r_{1}+r_{2}+\cdots+r_{k}+\cdots=1, \\
r_{k}>r_{k+1}+r_{k+2}+\cdots,
\end{gathered}
$$

the last one corresponding to $\xi_{k}<1 / 2$.

The points of the set are given by

$$
x=2 \pi\left[\epsilon_{1} r_{1}+\epsilon_{2} r_{2}+\cdots+\epsilon_{k} r_{k}+\cdots\right] \quad\left(\epsilon_{i}=0 \text { or } 1\right) .
$$

Now choose the $r_{k}$ 's in the following way: let $m_{1} m_{2} \cdots m_{n} \cdots$ be an increasing sequence of positive integers and let

$$
1 / 3^{m_{1}}+1 / 3^{m_{2}}+\cdots+1 / 3^{m_{n}}+\cdots=\gamma .
$$

We have $\gamma \leqq 1 / 3+1 / 3^{2}+\cdots=1 / 2$. Take

$$
r_{k}=(1 / \gamma) \cdot\left(1 / 3^{m_{k}}\right)
$$

Thus the first condition (12) is satisfied. The second also, since

$$
1 / 3^{m_{k}}>\left(1 / 3^{m_{k}}\right)\left[1 / 3+1 / 3^{2}+\cdots\right] \geqq 1 / 3^{m_{k+1}}+1 / 3^{m_{k+2}}+\cdots .
$$

Thus we have a set $P$ for which

$$
x=(2 \pi / \gamma)\left[\epsilon_{1} / 3^{m_{1}}+\epsilon_{2} / 3^{m_{2}}+\cdots+\epsilon_{n} / 3^{m_{n}}+\cdots\right] .
$$

This set is contained in $(0,2 \pi)$ and is homothetic to the set $E$ of the points $y$ such that

$$
y=4 \pi\left[\epsilon_{1} / 3^{m_{1}}+\epsilon_{2} / 3^{m_{2}}+\cdots+\epsilon_{n} / 3^{m_{n}}+\cdots\right],
$$

which is also contained in $(0,2 \pi)$. Now $E$ is a subset of the Cantor ternary set whose points are given by

$$
4 \pi\left[\epsilon_{1} / 3+\epsilon_{2} / 3^{2}+\cdots+\epsilon_{q} / 3^{q}+\cdots\right] ;
$$

hence $E$ is a set of uniqueness, and $P$ is a set of uniqueness by homothety. 
Now in this case we have

$$
\xi_{k} /\left(1-\xi_{k}\right)=\left(r_{k+1}+r_{k+2}+\cdots\right) / r_{k}=3^{m_{k}}\left(1 / 3^{m_{k+1}}+1 / 3^{m_{k+2}}+\cdots\right)
$$

which is a transcendental number if $\gamma$ is transcendental. Thus the $\xi_{k}$ can all be transcendental.

Besides, we have

$$
\lim \left(\xi_{1} \cdots \xi_{k}\right)^{1 / k}=\lim r_{k}^{1 / k}=\lim 3^{-m_{k} / k}
$$

and we can choose $m_{k}$ such that this limit be any number we like (less than $1 / 3)$. We can take, for example, $m_{k}=[k \alpha], \alpha$ being an irrational greater than 1 . This shows that in the theorem of $\$ 10$, the hypothesis $\lim \xi_{p}=\xi$ can not be replaced by $\lim \left(\xi_{1} \xi_{2} \cdots \xi_{p}\right)^{1 / p}=\xi$.

We observe that we can have $\gamma$ transcendental with a sequence $\left\{m_{k}\right\}$ of arbitrary positive density. For if $\gamma$ were not transcendental we can add to it a Liouville number of the form $\sum 3^{-n !}$, which does not alter the density of the sequence $\left\{m_{k}\right\}$.

13. A sufficient condition for a symmetrical perfect set to be a set of uniqueness. In the preceding example we have constructed a symmetrical perfect set of uniqueness for which the arithmetic nature of the $\xi_{k}$ was arbitrary but the $\xi_{k}$ were all connected in a simple way with the same number $\gamma$.

In the following theorem, we shall get a set of uniqueness by imposing on the $\xi_{k}$ conditions related only with their order of magnitude, but in no way with their arithmetical properties.

THEOREM. If there exists an infinite sequence $S$ of integers $q$ such that

$$
\left(\xi_{1} \xi_{2} \cdots \xi_{q}\right)^{1 / q} \leqq \alpha / q
$$

and if for the same sequence of integers $q$ we have, for a positive $\delta$,

$$
\xi_{q+1} \leqq 1 / 2-2 \alpha-\delta
$$

then the set is of the type $H$, hence a set of uniqueness.

The numbers $r_{k}$ having the same signification as above, we have for every $x$ belonging to the set

$$
x=2 \pi\left[\epsilon_{1} r_{1}+\epsilon_{2} r_{2}+\cdots+\epsilon_{k} r_{k}+\cdots\right] \quad\left(\epsilon_{i}=0 \text { or } 1\right) .
$$

Let $q$ be a number of the sequence $S$. By Dirichlet's theorem we can find an integer $n=n(q) \leqq(q / \alpha)^{q}$, increasing infinitely with $q$, and such that:

We have

$$
\left|\left\{n r_{1}\right\}\right| \leqq \alpha / q,\left|\left\{n r_{2}\right\}\right| \leqq \alpha / q, \cdots,\left|\left\{n r_{q}\right\}\right| \leqq \alpha / q .
$$

$$
\begin{aligned}
n x= & 2 \pi\left[\epsilon_{1} A_{1}+\epsilon_{2} A_{2}+\cdots+\epsilon_{q} A_{q}\right]+2 \pi\left[\epsilon_{1}\left\{n r_{1}\right\}+\cdots+\epsilon_{q}\left\{n r_{q}\right\}\right] \\
& +2 \pi\left[\epsilon_{q+1} n r_{q+1}+\epsilon_{q+2} n r_{q+2}+\cdots\right]
\end{aligned}
$$


or

$$
n x=N(x)+\alpha(x)+y(x),
$$

say, where $N(x), \alpha(x), y(x)$ denote the three successive terms in the second member of (13). The $A_{i}$ are integers, and we can write

$$
n x \equiv \alpha(x)+y(x)(\bmod 2 \pi) \text {. }
$$

We have

$$
|\alpha(x)| \leqq 2 \pi q \alpha / q=2 \pi \alpha .
$$

We have also

$$
n \xi_{1} \cdots \xi_{q} \leqq n(\alpha / q)^{q} \leqq 1
$$

and

$$
\begin{aligned}
y(x) & =n \xi_{1} \cdots \xi_{q} \cdot 2 \pi\left[\epsilon_{q+1}\left(1-\xi_{q+1}\right)+\epsilon_{q+2} \xi_{q+1}\left(1-\xi_{q+2}\right)+\cdots\right] \\
& =n \xi_{1} \cdots \xi_{q} \cdot z(x),
\end{aligned}
$$

say, where $z(x)$ denotes a point belonging to the symmetrical perfect set constructed on $(0,2 \pi)$ with the sequence $\left(\xi_{q+1}, \xi_{q+2}, \cdots\right)$. This symmetrical perfect set has a contiguous interval of length $2 \pi\left(1-2 \xi_{q+1}\right)$. Thus the set of points $y$, which lies entirely in $(0,2 \pi)$, has a contiguous interval of length $2 \pi n \xi_{1} \cdots \xi_{q}\left(1-2 \xi_{q+1}\right)$. But the set of points $y$ has also, in $(0,2 \pi)$, a contiguous interval of length $2 \pi-2 \pi n \xi_{1} \cdots \xi_{q}$ since

$$
y(x) \leqq 2 \pi n \xi_{1} \cdots \xi_{q} \text {. }
$$

Hence the set of points $y$ has a contiguous interval of length $\lambda$ not less than

$$
\max \left\{2 \pi n \xi_{1} \cdots \xi_{q}\left(1-2 \xi_{q+1}\right), 2 \pi\left(1-n \xi_{1} \cdots \xi_{q}\right)\right\}
$$

hence not less than

$$
\frac{2 \pi n \xi_{1} \cdots \xi_{q}\left(1-2 \xi_{q+1}\right)+2 \pi\left(1-n \xi_{1} \cdots \xi_{q}\right)\left(1-2 \xi_{q+1}\right)}{2-2 \xi_{q+1}},
$$

that is to say not less than

$$
\pi\left(1-2 \xi_{q+1}\right) /\left(1-\xi_{q+1}\right)>\pi\left(1-2 \xi_{q+1}\right) .
$$

Now we have by our hypothesis

hence

$$
1-2 \xi_{q+1} \geqq 4 \alpha+2 \delta
$$

$$
\lambda \geqq 4 \pi \alpha+2 \pi \delta .
$$

Now by (15) $|\alpha(x)| \leqq 2 \pi \alpha$. Hence by (14) we see that the set of points $n x$, reduced modulo $2 \pi$, has a contiguous interval of length not less than

$$
\lambda-2 \max |\alpha(x)| \geqq \lambda-4 \pi \alpha \geqq 2 \pi \delta \text {. }
$$


Thus the set of points $x$ is of the type $H$, which proves the theorem.

In particular we see that the set is a set of uniqueness if

$$
\lim \inf q\left(\xi_{1} \cdots \xi_{q}\right)^{1 / q}=0 \text { and } \lim \sup \xi_{q}<1 / 2,
$$

more particularly if

$$
\xi_{q}=o(1 / q) \text {. }
$$

\section{Bibliography}

R. SALEM

1. Sets of uniqueness and sets of multiplicity, Trans. Amer. Math. Soc. vol. 54 (1943) pp. 218-228.

2. On sets of multiplicity for trigonometrical series, Amer. J. Math. vol. 64 (1942) pp. 531538.

3. A remarkable class of algebraic integers. Proof of a conjecture of Vijayaraghavan, Duke Math. J. vol. 11 (1944) pp. 103-108.

Massachusetts Institute of Technology,

Cambridge, Mass.

HARVARD UNIVERSITY,

Cambridge, Mass. 